\title{
NASA panel weighs asteroid danger
}

Telescopes in space could help pin down the risk of a deadly impact.

Some time in the next decade, a US president will probably be presented with this dilemma: is it worth spending US $\$ 1$ billion to deflect a space rock that may never hit Earth?

A NASA panel is wrestling with this question, which is growing more pertinent as scientists' ability to find asteroids that pose a potential risk, termed near-Earth objects (NEOs), outstrips their capacity to track them accurately. The Ad-Hoc Task Force on Planetary Defense, set up to suggest ways for the agency to protect Earth against a deadly impact, is expected to release its report next month. But public delib-

Missions to deflect all potential threats will be prohibitively expensive. erations and interviews with its members have revealed their thinking.

The dilemma stems from a 2005 congressional mandate directing NASA to $\log 90 \%$ of the estimated 20,000 NEOs larger than 140 metres in diameter by 2020 . NASA seems unlikely to meet the goal, but the agency is stepping up its detection and tracking of smaller objects.
That will create a new problem: if the pace of NEO detections (see graph) grows but precision tracking of orbits lags behind, observers will start to find more rocks - perhaps a few per year that seem, at first, to have a significant chance of hitting Earth, say panel members. "I don't think that issue has been understood outside the NEO community," says Lindley Johnson, NEO programme officer at NASA and a member of the panel. Launching missions to track or deflect all potential asteroid threats will be prohibitively expensive, but even a small probability of regional or global devastation may not be politically palatable.

One solution from the panel is to increase the amount that the United States invests in NEO detection and tracking from the current $\$ 5.5$ million a year. The panel may also recommend the launch of a survey telescope into a solar orbit similar to that of Venus. It would orbit faster than Earth and, looking outwards, would see asteroids in Earth-crossing orbits more often than would ground-based instruments (see diagram). This could improve follow-up observations, narrow estimated trajectories and remove as many asteroids as possible from the threat list. It could also spot and track asteroids on the sunward side of Earth, removing a worrisome blind spot in ground-based surveys. "It is a wonderful rapid technique to track bodies down to 140 metres and smaller," says Tom Jones, a former astronaut and panel co-chair.

Ball Aerospace and Technologies Corporation, a manufacturer of spacecraft based in Boulder, Colorado, has proposed building such a remote scope at a cost of $\$ 600$ million. But Irwin Shapiro, an astrophysicist at the HarvardSmithsonian Center for Astrophysics in Cambridge, Massachusetts, who chaired the 2010 Committee to Review Near-Earth-Object Surveys and Hazard Mitigation Strategies for the US National Research Council, says that groundbased observatories such as the planned Large Synoptic Survey Telescope (LSST) on Cerro Pachón in Chile are better value for money

\section{Rare victory in fight against melanoma}

Patients with advanced melanoma rarely live for more than a year after their diagnosis - a prognosis that has not improved for more than 30 years. But clinical-trial results ${ }^{1}$ now suggest that a genetically targeted approach could slow the disease's steady march through the body, and separate research ${ }^{2}$ reveals why the latest drug being tested may succeed where others failed.

The drug could be the first melanoma treatment to join an elite but expanding list of cancer therapies tailored to a patient's genetic make-up (see 'Seek and destroy'). "It's spectacular," says Richard Marais, a molecular biologist at the Institute of Cancer Research in London, who was not affiliated with either study. "There's been a paradigm shift in how you would treat melanoma."

In 2002, Marais and his colleagues reported ${ }^{3}$ that more than $60 \%$ of patients with melanoma cancer of the melanin-producing cells typically found in skin - carry mutations in a gene that encodes a protein called B-RAF. The mutations trigger a signalling pathway that accelerates cancerous cell growth.

Drug companies pricked up their ears at the discovery. One of them, a small biotechnology company in Berkeley, California, called Plexxikon, started fishing for compounds that would selectively block mutated B-RAF by filling the unique pocket that the genetic mutation etches into the protein's three-dimensional structure.

At first, the approach was greeted with scepticism, says Plexxikon's chief executive Peter Hirth. Decades of failure had taught drug companies to shy away from testing drugs against advanced melanoma. "People don't want to do clinical trials in melanoma," agrees Marais. "It's sort of the death knell for all cancer therapies."

Early tests of Plexxikon's drug, called PLX4032, were
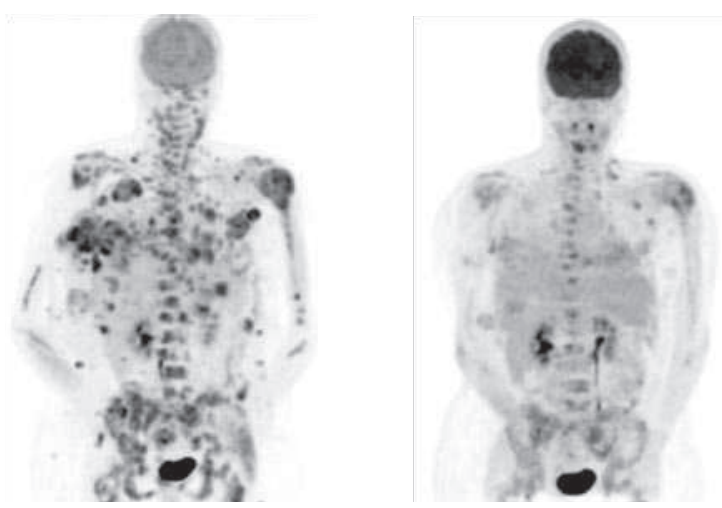

Melanoma tumours shrank after patients took PLX4032 for 2 weeks.

disappointing. Some patients in the trial were taking dozens of pills a day, to no effect. "But we just couldn't give the patients any more pills," says Paul Chapman, a cancer researcher at the Memorial SloanKettering Cancer Center in New York and a co-author on the latest clinical trial'. "We were starting to think we were going to obstruct their gut with all the cellulose from the capsules." So Plexxikon, together with its partner, Swiss drug maker Roche, developed a new formulation of the drug that was more easily absorbed by the body.

That reformulation proved crucial. On 26 August, researchers published the first findings from a small clinical trial of the reformulated drug. In one arm of the study ${ }^{1}$, tumours shrank by at least 


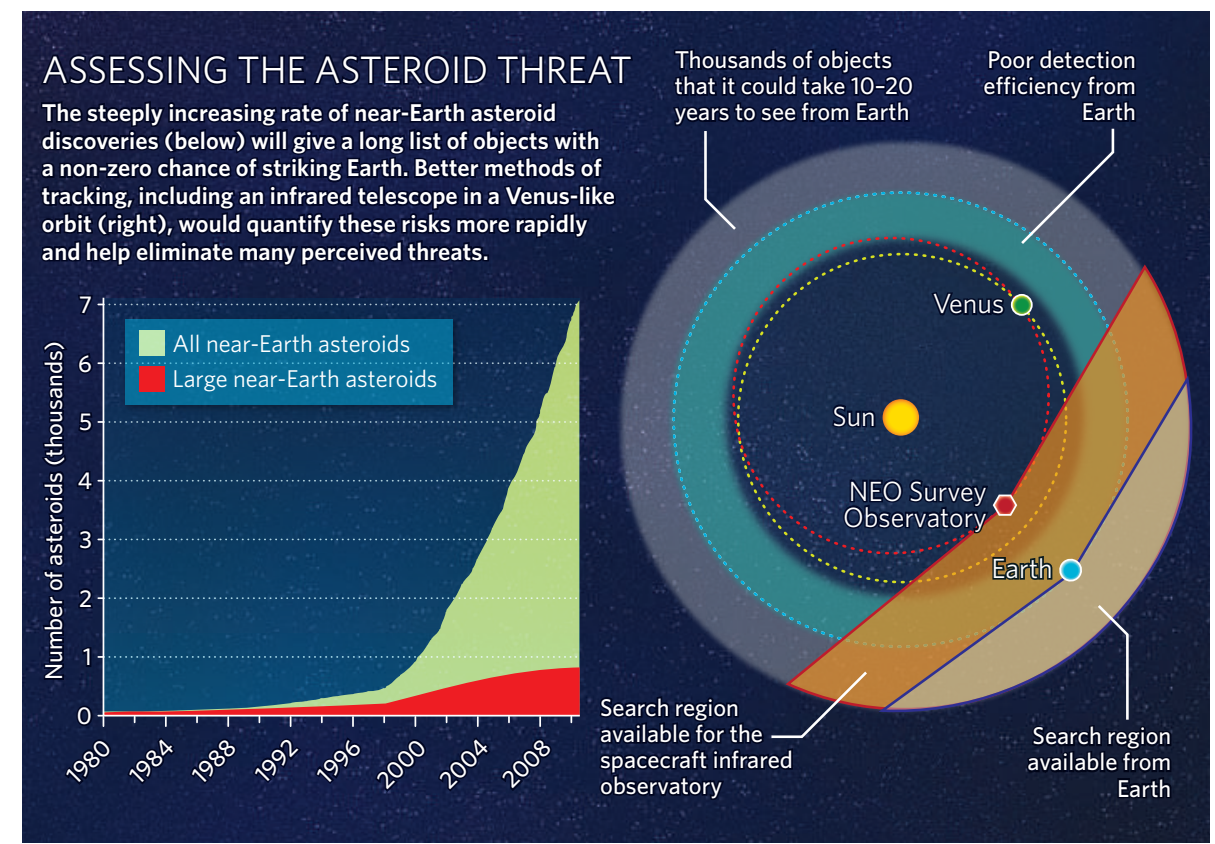

than space telescopes, because they last longer and are less expensive. He says the LSST is also more likely to command funding, as it is the top priority recommended by the Astronomy and Astrophysics Decadal Survey, released in August by the National Academies. Putting a space telescope in a Venus-like orbit "would in effect start from scratch", he says.

Owing to a 2008 law passed by Congress, the White House Office of Science and Technology Policy has until 15 October to decide which agency will be responsible for protecting the planet from an asteroid strike. Members of the task force say NASA expects to be given part or all of that responsibility. To meet it, the panel discussed the creation of a Planetary Protection Coordination Office (PPCO) within NASA, with an annual budget of $\$ 250$ million-\$300 million. It would detect and track asteroids - and develop a capability to deflect them. "You want to use a proven capability when you're talking about an actual threat," says Rusty Schweickart, a former astronaut and the other panel co-chair.

The PPCO would also challenge other countries to fund defence against asteroids, perhaps through the United Nations. Canada already plans to launch the NEO Survey Satellite in 2011, and Germany's AsteroidFinder is slated for launch in 2012, but neither is expected to come close to the NEO-logging goal by 2020.

Shapiro stresses that it is unclear whether Congress will give further funds to planetary protection, noting that if it doesn't, there is a risk of the money being taken away from space science. Yet without better detection and tracking there will inevitably be uncertainty about asteroid positions in the future - and even greater expense if the uncertainty leads to unnecessary efforts to thwart an apparent pressing threat. Eugenie Samuel Reich
$30 \%$ in 24 out of 32 patients with B-RAF mutations, and disappeared entirely in two other patients.

This week in Nature ${ }^{2}$, the Plexxikon team reveals why the high doses were necessary: analysis of tumour samples from patients who received different amounts of the drug showed that PLX4032 was effective only at concentrations sufficient to block about $80 \%$ of signalling through the B-RAF pathway. This roughly matches early clinical-trial results of a similar drug being developed by Londonbased GlaxoSmithKline (GSK), says Jeffrey Legos, the company's medicines development leader.

The Plexxikon team also found that PLX4032 was remarkably specific for the mutated form of B-RAF, binding relatively poorly, if at all, to more than $\mathbf{2 0 0}$ other proteins, including unmutated B-RAF. That selectivity, says Hirth, probably explains why patients were able to take PLX4032 at such high doses without severe side effects.

Despite the promising results, PLX4032 has been tested in only a small number of patients and it is too early to declare the drug a success, cautions Chapman. The next stage is a larger trial in 700 patients, which began in January.

And although PLX4032 has clear short-term effects on tumour growth, researchers don't yet know whether the drug will allow patients to live significantly longer. Melanoma tumours quickly become resistant to PLX4032, and

\section{Seek and destroy}

Cancer patients who have a particular genetic profile could benefit from a small but growing family of drugs.

\begin{tabular}{|c|c|c|}
\hline $\begin{array}{l}\text { TRASTUZUMAB } \\
\text { Brand name: } \\
\text { Herceptin } \\
\text { Sold by: Roche } \\
\text { (Genentech) } \\
\text { Cancer: breast } \\
\text { Mutation: } \\
\text { amplification of the } \\
\text { HER2 gene } \\
\text { Protein targeted: } \\
\text { HER2/neu receptor }\end{array}$ & $\begin{array}{l}\text { IMATINIB } \\
\text { Brand name: Gleevec } \\
\text { Sold by: Novartis } \\
\text { Cancer: chronic } \\
\text { myeloid leukaemia, } \\
\text { others } \\
\text { Mutation: fusion of } \\
\text { BCR and ABL genes } \\
\text { Protein targeted: } \\
\text { several tyrosine } \\
\text { kinases, including } \\
\text { Bcr-Abl }\end{array}$ & $\begin{array}{l}\text { ERLOTINIB } \\
\text { Brand name: Tarceva } \\
\text { Sold by: Roche } \\
\text { (Genentech) and OSI } \\
\text { Pharmaceuticals } \\
\text { Cancer: non-small-cell } \\
\text { lung cancer, others } \\
\text { Mutation: epidermal- } \\
\text { growth-factor } \\
\text { receptor (EGFR) gene } \\
\text { Protein targeted: } \\
\text { EGFR tyrosine kinase }\end{array}$ \\
\hline
\end{tabular}

PLX4032 will be tested in combination with other drugs. In addition to the larger trial of PLX4032 alone, Plexxikon is planning a trial that will combine PLX4032 with an experimental drug that blocks MEK, another protein involved in the cell-growthpromoting pathway. Meanwhile, GSK is recruiting patients for a clinical trial combining two of its own experimental drugs, which respectively block mutated B-RAF and MEK.

For now, the promising results from PLX4032 highlight the power of targeting specific genetic mutations in tumours, says Yardena Samuels, a cancer geneticist at the National Human Genome Research Institute in Bethesda, Maryland. "It's a very important development, not just for melanoma, but for the entire cancer field."

Heidi Ledford

1. Flaherty, K. T. et al. N. Engl. J. Med. $\mathbf{3 6 3}$, 809-819 (2010).

2. Bollag, G. et al. Nature doi:10.1038/ nature09454 (2010).

3. Davies, H. et al. Nature $417,949-954$ (2002). 